\title{
Valutazione del nuovo sistema automatizzato Triturus - Grifols nella diagnosi sierologica delle infezioni da complesso ToRCH e da virus di Epstein Barr
}

\author{
Monica Sparacino', Maria Antonella Simone', Gian Matteo Pollini', Vittorio Sambri', \\ Roberto Cevenini' \\ ' Sezione di Microbiologia, DMCSS, Università di Bologna; \\ ${ }^{2}$ Unità Operativa Microbiologia settore batteriologia speciale e sierologia, Azienda Ospedaliera di Bologna, Ospedale \\ S.Orsola, Bologna, Italia.
}

Evaluation of the Triturus- Grifols automated system in the serological diagnosis of ToRCH and EBV infections

Key Words: EIA, Cytomegalovirus, Rubella virus, Herpes simplex virus, Epstein Barr virus, Toxoplasma gondii.

\section{SUMMARY}

The laboratory diagnosis of infections belonging to the ToRCH complex is mainly based of the determination of the immune status to each individual microrganism. In this report, the diagnostic performance of a new automated EIA plates processor system (SeraQuest - GRIFOLS, Pisa, Italy) has been compared with other automaed methods: Enzygnost (Dade behring, Marburg, Germany), EIA Well (RADIM, Pomezia, Italy), VIDAS (bioMériuex, Marcy l'etoile, France). In addition, the diagnostic performance of SeraQuest has also been evaluated for the detection of specific immune response to Epstein Barr virus, in comparison with a conventional ElA test (Delta, Pomezia, Italy) and with an immunoblotting method (Genelabs - AlfaBiotech, Milan, Italy). The overal evaluation of SeraQuest demonstrated that this test has sensitivity and specificity well comaparable with those of the other methods studied and that could be used as an alternative test in the serological diagnosis of ToRCH and EBV infections.

\section{INTRODUZIONE}

Lo scopo di questo studio è stato quello di presentare i risultati qualitativi ottenuti col nuovo sistema analitico Triturus-Grifols per la diagnosi sierologica di patologie ad eziologia infettiva. Tale sistema si basa sulla esecuzione di saggi immunoenzimatici su micropiastra (linea SeraQuest-GRIFOLS), eseguiti in automatico (con analizzatore automatico Triturus). Allo scopo di discutere le caratteristiche del sistema in studio si sono paragonati i risultati ottenuti col presente metodo con quelli ricavati da altri sistemi commerciali noti per affidabilità e riproducibilità dei risultati $(1,2,9,11)$, quali il sistema VIDAS della bioMérieux (rappresentante di un sistema analitico cosiddetto "chiuso"), i test ELISA-Radim automatizzati sullo strumento ARIO e i kit Dade Behring automatizzati su analizzatore BEP III (questi ultimi due kits sono stati considerati rappresentanti di sistemi analitici "aperti").

Le patologie di cui si indaga la risposta sierologica sono causate da agenti microbici facenti parte del complesso ToRCH (Toxoplasma gondii, Rubeovirus, Citomegalovirus ed Herpes Simplex virus) e dal virus di Epstein Barr. Il
Citomegalovirus (CMV) è un virus erpetico e il suo effetto sulle cellule si caratterizza per il rigonfiamento delle cellule ospiti e per la formazione di inclusi citoplasmatici e nucleari. Oggi è considerato tra i microorganismi più importanti nel provocare anomalie congenite in seguito ad infezioni intra-uterine, inoltre si ritiene essere responsabile di complicazioni nel paziente sottoposto a trapianto d'organo, in particolare in caso di trapianto di rene e di trapianto ortotopico cardiaco. L'infezione da CMV è largamente diffusa nella popolazione e spesso sottostimata poichè asintomatica. Gli anticorpi anti CMV si rilevano nel 50$70 \%$ dei giovani adulti: negli individui immunocompetenti le IgM prodotte in seguito alla infezione primaria solitamente persistono dai 2 ai 9 mesi. Nei pazienti trapiantati e negli immunodepressi gli anticorpi IgM possono persistere più a lungo (7) (anche per periodi di oltre 2 anni). Il virus della rosolia (Rubeovirus) appartiene al gruppo dei Togavirus: normalmente la rosolia, acquisita dopo la nascita, si manifesta come una malattia autolimitantesi, a decorso totalmente benigno che lascia nell'ospite come unica traccia un'immunità duratura protettiva per le successive infezioni. Se la rosolia viene contratta in gravi- 
danza e in particolare nel primo trimestre, pur non provocando alcun danno clinicamente evidente alla gestante, può avere degli effetti devastanti sull'embrione e sul feto: si ha la comparsa di lesioni cardiovascolari, sordità, corioretinite, ritardo mentale e della crescita in genere (12). La determinazione dello stato immune IgG, provocata sia dall'infezione naturale sia dalla stimolazione vaccinale, resta un momento fondamentale nella diagnosi e nella eventuale profilassi di questa infezione. La determinazione della risposta IgM viene invece considerata un utile ausilio diagnostico per la definizione delle fasi attive dell'infezione. Il protozoo Toxoplasma gondii è un parassita endocellulare e provoca infezioni sia nell'uomo che negli animali. L'infezione da $T$. gondii è piuttosto comune e le cause più frequenti sono il consumo di carne cruda o poco cotta contaminata o il contatto occasionale con deiezioni di animali infetti. L'andamento dell'infezione generalmente è tenue o subclinico: sia nell'adulto che nell'adolescente si evidenzia con sintomi quali linfoadenopatia, febbre, linfocitosi e mialgie. La corioretinite è una manifestazione della forma acquisita, ma può anche trattarsi di una recidiva a comparsa ritardata della forma congenita. I soggetti più vulnerabili all'infezione sono gli immunodepressi (in particolare le encefaliti da Toxoplasma sono comuni tra i pazienti affetti da AIDS (3)) e il feto, soprattutto nel primo semestre di gravidanza (5). Durante questo periodo di gravidanza il SNC è in fase di sviluppo e l'esito dell'infezione verticale da $T$. gondii è spesso infausto con esito in aborto oppure, in caso di prosecuzione della gestazione si ha la possibile comparsa nella successiva vita extrauterina di gravi anomalie quali corioretiniti, calcificazioni intracerebrali, disturbi psicomotori, idrocefalo e microcefalia. Il virus di Epstein Barr appartiene alla famiglia dei virus erpetici ed è un patogeno umano ubiquitario. Infatti circa il $90 \%$ della popolazione adulta presenta anticorpi specifici nei confronti del virus. Dopo l'infezione primaria che può presentarsi come mononucleosi infettiva, l'EBV persiste in forma latente integrato nelle cellule linfocitarie dell'ospite e in situazioni di immunocompromissione può riattivarsi dando luogo, in particolare, a patologia linfoproliferativa (linfomi). Durante l'infanzia l'infezione primaria da EBV passa spesso inosservata perchè asintomatica, mentre in età postpuberale da luogo alla forma clinicamente evidente di mononucleosi infettiva, caratterizzata da faringite, febbre elevata, linfoadenopatia cervicale o diffusa ed epato-splenomegalia (6).

La diagnosi delle infezioni causate dagli agenti microbici appartenenti al complesso ToRCH descritti è frequentemente ottenuta sulla base del dato sierologico che diventa necessario anche in casi di diagnosi differenziale tra malattie isosintomatiche. Il dato di laboratorio sierologico vede la sua principale applicazione però nella diagnosi di stato immune, condizione che ha notevolissima importanza per definire i rischi correlati alle infezioni del complesso ToRCH in gravidanza (10). Il dosaggio degli anticorpi di classe IgM (14) indica un'infezione in atto o molto recente, mentre la presenza di anticorpi sierici di classe IgG può essere utile in fase di screening prenatale per il complesso ToRCH (4). Infatti il feto durante la gravidanza, in caso di madre non immune per uno qualsiasi degli agenti infettivi sopra menzionati, sarà esposto ad un rischio di infezione, con percentuali differenti a seconda del patogeno considerato e della fase di gestazione.

\section{MATERIALI E METODI SeraQuest (GRIFOLS)-TRITURUS}

La linea SeraQuest ToRCH ed EBV della Grifols diagnostic, propone un pannello omogeneo di prodotti con metodologia ELISA per la determinazione della risposta immune di classe $\mathrm{IgG}$ e IgM nei confronti dei patogeni del complesso ToRCH e nei confronti di antigeni specifici di EBV. Per tutti si tratta di un test ELISA su micropiastra a 96 pozzetti, uniforme ed omogeneo come procedimento analitico, reattivi ed accessori comuni e caratteristiche qualitative simili. Il principio metodologico è quello di un dosaggio immunoenzimatico: l'antigene adeso alle fase solida (micropiastra) viene riconosciuto dagli anticorpi specifici presenti nel siero del paziente e questi a loro volta sono riconosciuti da anticorpi anti-IgG specifici o anti-IgM umane, ottenuti in animale e coniugati all'enzima rivelatore (perossidasi), che agendo su un opportuno substrato cromogeno induce un cambiamento di colore che, opportunamente misurato e comparato con quanto avviene coi sieri calibratori del metodo, permette una valutazione semiquantitativa del legame antigene/anticorpo. Dal confronto con i valori dei due calibratori, del controllo positivo e del controllo negativo forniti nel kit viene calcolato il risultato (positivo, negativo o dubbio) di ogni campione.

L'apparecchio denominato Triturus è un analizzatore walk-away ad elevate prestazioni per la gestione completamente automatica dei test con metodologia ELISA capace di adattarsi alle diverse realtà operative del laboratorio di sierologia. Gestisce auomaticamente 4 piastre microtiter a 96 pozzetti, indipendenti per temperatura di incubazione ed eventuale agitazione. Dispone di un carosello portacampioni per 92 provette, con identificazione positiva mediante codice a barre. 
Permette l'esecuzione automatica di pannelli di test fino ad un massimo di 8 metodiche in contemporanea. Il tempo di esecuzione della seduta varia da un minimo di 1 ora e 45 minuti ad un massimo di 3 ore e 15 minuti quando all'interno della stessa seduta vengano analizzati il massimo numero di campioni su diversi parametri analitici. A seduta già iniziata è possibile inserire un nuovo test, un nuovo campione o un nuovo batch, questo grazie alla capacità multitasking del software di gestione. Il software che gestisce il sistema automatico TRITURUS è risultato di facile comprensione e gestione intuitiva da parte del personale; il tempo di preparazione delle sedute non ha mai superato i 20 minuti (anche in caso di numero massimo di campioni processabili simultaneamente). Il Triturus è ideale sia per le "grandi routine", grazie alla possibilità di sostituire il carosello portacampioni non appena sia terminata la fase di dispensazione dei campioni diluiti in piastra; sia per le "piccole routine" dal momento che è possibile utilizzare solo i pozzetti realmente necessari al dosaggio e lavorare a batch di numerosi parametri con pochi campioni per ciascun parametro, con uno spreco minimo di reattivi e di materiali.

\section{EIA well - Radim}

Il kit della linea Radim utilizzato come termine di paragone per la diagnostica dell'infezione da Toxoplasma gondii è basato sul metodo immunoenzimatico (ELISA). Il test EIA well è stato eseguito su di un analizzatore automatico ARIO (Seac-RADIM) secondo le istruzioni del produttore.

\section{VIDAS bioMériuex}

Il metodo VIDAS - bioMériuex usato nello studio come test di confronto per la diagnosi di infezione da Toxoplasma gondii, virus della Rosolia e Citomegalovirus, si basa sulla metodica ELFA (Enzyme-linked immunofluorescent assay).

Il principio del dosaggio associa il metodo immunoenzimatico ad una rivelazione finale in fluorescenza (ELFA) ed è stato impiegato sullo strumento automatico dedicato secondo le indicazioni del Produttore.

\section{Enzygnost Dade Behring}

Il sistema Dade Behring si basa sull'impiego dell'analizzatore semi-automatico BEP III che processa le micropiastre di un metodo immunoenzimatico per l'identificazione qualitativa e la determinazione quantitativa degli anticorpi umani. È stato utilizzato in queso studio come test di riferimento per la diagnosi sierologica di infezione da virus della Rosolia, Citomegalovirus e virus di
Epstein Barr.

Le determinazioni sono state eseguite mediante l'impiego di un diluitore GENESIS RSP100 per la preparazione delle micropiastre secondo il software e le indicazioni fornite dal Produttore.

\section{Immunowell - Delta Biologicals}

Il kit Delta Biologicals per la determinazione della risposta immune verso antigeni specifici del virus di Epstein Barr è basato su un metodo ELISA per la determinazione qualitativa o semiquantitativa degli anticorpi anti-VCA IgG e antiVCA IgM presenti nel siero. Anche questo test è stato eseguito secondo le indicazioni del Produttore.

\section{Western Blot EBV - Genelab}

Al fine di estendere la valutazione della performance diagnostica del sistema TRITURUS è stato impiegato, per confronto, anche un test basato sulla tecnica di western blot (Genelabs) per la determinazione degli anticorpi IgM e IgG verso il virus di Epstein Barr.

In questo caso il kit si basa sulla classica metodica dell'immunoblotting: dopo separazione elettroforetica di 4 differenti antigeni ricombinanti di EBV (VCA p23; EA diffuse p54; EA p138 e EBNA-1 p72) vengono trasferiti su strisce di nitrocellulosa che vengono incubate ognuna con un siero in esame.

Il legame fra anticorpi sierici e singoli antigeni di EBV viene rivelato con anticorpi di coniglio antiIg umane coniugati con fosfatasi alcalina, che induce la catalisi enzimatica del substrato.

L'interpretazione dei risultati avviene mediante analisi del pattern delle bande antigeniche identificata da ciascun siero.

\section{Sieri studiati}

Nel nostro studio abbiamo preso in considerazione diversi campioni di sieri suddivisi per i differenti antigeni secondo lo schema riportato in tabella 1. Nel dettaglio sono stati testati: 30 sieri per valutare la presenza di anticorpi IgG e 30 per valutare la presenza di anticorpi IgM contro $T$. gondii; 40 sieri per ricercare anticorpi $\operatorname{IgG}$ e 30 per ricercare anticorpi IgM contro Rubeovirus; 35 sieri per determinare anticorpi IgG e 35 per determinare anticorpi IgM contro Citomegalovirus, infine 40 sieri per la ricerca di anticorpi IgG e 51 sieri per anticorpi IgM contro EBV. I campioni sono stati selezionati in base ai risultati ottenuti dai tests eseguiti sui sistemi di diagnosi sierologica in uso nei nostri laboratori: nel dettaglio, per la selezione di sieri da indagare per anticorpi contro T. gondii si è fatto riferimento ai risultati ottenuti col sistema Radim-Ario, mentre per quanto con- 
cerne i sieri scelti per testare anticorpi contro $\mathrm{CMV}, \mathrm{EBV}$ e Rubeovirus ci si è basati sui risultati ottenuti col sistema Dade Behring-Genesis BEP III.

L'obiettivo del lavoro è stato quello di confrontare (13) in termini di concordanza, sensibilità e specificità il sistema analitico Seraquest fornito da Grifols sul Triturus con le diverse metodiche ELISA della concorrenza.

Tabella I. Numero di campioni saggiati e relative metodiche impiegate

\begin{tabular}{lll}
\hline PARAMETRO & N. CAMPIONI TESTATI & SISTEMI UTILIZZATI \\
\hline T. gondii IgG & 30 & SeraQuest su Triturus \\
& & EIA well Radim su ARIO \\
& bioMérieux su VIDAS \\
\hline T. gondii IgM & 30 & $\begin{array}{l}\text { SeraQuest su Triturus } \\
\text { EIA well Radim su ARIO } \\
\text { bioMérieux su VIDAS }\end{array}$ \\
\hline Rubella IgG & 40 & SeraQuest su Triturus \\
& & Enzygnost Dade Behring su BEP III \\
& & bioMérieux su VIDAS \\
\hline Rubella IgM & 30 & SeraQuest su Triturus \\
& & Enzygnost Dade Behring su BEP III \\
& bioMérieux su VIDAS \\
\hline CMV IgG & 35 & SeraQuest su Triturus \\
& & Enzygnost Dade Behring su BEP III \\
& bioMérieux su VIDAS \\
\hline CMV IgM & 35 & SeraQuest su Triturus \\
& & Enzygnost Dade Behring su BEP III \\
& bioMérieux su VIDAS \\
\hline EBV IgG & SeraQuest su Triturus \\
& Enzygnost Dade Behring su BEP III \\
& Immunowell Delta Biologicals. \\
& Western blot EBV Genelab \\
\hline EBV IgM & 5 SeraQuest su Triturus \\
& & Enzygnost Dade Behring su BEP III \\
& Immunowell Delta Biologicals \\
& Western blot EBV Genelab \\
\hline &
\end{tabular}

\section{RISULTATI}

I dati comparativi ottenuti per la determinazione di anticorpi sierici anti $T$. gondii (IgG e IgM) su 30 campioni, mediante l'impiego delle metodiche GRIFOLS, RADIM, bioMérieux mostrano come il numero di campioni IgG positivi identificati con le metodiche GRIFOLS e RADIM sia equivalente (22 e 23 positivi rispettivamente), mentre per la metodica ELFA-VIDAS tale numero risulta di poco più alto ( 25 positivi). Per la determinazione delle IgM anti T. gondii il numero dei positivi risulta sovrapponibile per le tre metodiche usate, così come identico è risultato il numero dei sieri identificati come IgM negativi. Tuttavia la metodica GRIFOLS riporta un campione borderline per le $\operatorname{IgM}$ in meno rispetto alle altre due metodiche.

Per quel che riguarda i risultati ottenuti sui 40 sieri testati con le metodiche GRIFOLS, Dade Behring e bioMérieux per la determinazione degli anticorpi IgG nei confronti di Rubeovirus, la metodica Dade Behring individua 33 positivi con- tro i 30 delle altre due e la riconferma di questa sensibilità leggermente più alta della tecnica Dade Behring si ha analizzando i dati dei 30 sieri studiati per le IgM. Infatti anche in questo caso il numero di sieri IgM positivi per Rubeovirus è di 23 contro i 17 positifivi identificati con la metodica GRIFOLS e i 19 determinati mediante la tecnica ELFA-VIDAS; i 6 sieri con valori IgM border line individuati dalla metodica GRIFOLS vengono di fatto identificati come positivi a basso titolo dalla tecnica Dade Behring.

Per quanto riguarda il Citomegalovirus (35 i sieri studiati) la concordanza per le IgG (29 positivi per GRIFOLS, 28 per Behring e per VIDAS) non è correlata ad un uguale valore di concordanza per le IgM: Dade Behring identifica 21 positivi, GRIFOLS 17 e bioMérieux 19 positivi e 16 negativi. Per il virus di Epstein Barr oltre a metodiche ELISA è stata utilizzata anche una metodica di immunoblotting che ha mostrato alcune interessanti differenze in termini di performance diagnostica rispetto al metodo EIA della GRIFOLS, impiegato per la stessa determinazione di risposta immune nei confronti di questo diffuso virus erpetico.

Si evidenzia così che il numero (su 40 sieri studiati per IgG e 51 per $\operatorname{IgM}$ ) di positivi identificati con questo test si avvicina di più, ma non raggiunge, il numero di campioni IgG positivi identificati dalle metodiche immunoenziamtiche di Dade behring e DELTA (rispettivamente 30 e 31). I dati dei sieri (n.51) testati per le IgM sono simili per la metodica EIA di GRIFOLS (18 positivi, 3 borderline e 30 negativi), di DELTA (17 positivi, 5 borderline e 29 negativi) ed anche per il metodo immunoblotting di GENELABS (20 positivi, 1 dubbio e 30 negativi). I risultati ottenuti con il test Enzygnost Dade Behring hanno permesso di individuare 13 campioni positivi, 23 sieri negativi ed una considerevole percentuale di campioni borderline (n.15).

La valutazione dei dati sopra elencati è stata eseguita calcolando i valori di specificità e sensibilità del metodo SeraQuest GRIFOLS in paragone con le altre metodiche impiegate, come riportato nella tabella 2 .

\section{DISCUSSIONE}

I dati sopra riportati depongono per una notevole specificità dei test SeraQuest GRIFOLS, quando valutati comparativamente con tre metodiche commercialmente disponibili e considerate come "riferimento" nel nostro laboratorio. Va sottolineato che tutti i test SeraQuest GRIFOLS hanno mostrato performances diagnostiche sovrapponibili a quelle dei test diagnostici valutati comparativamente di identica filosofia costruttiva (micro- 
Tabella 2. Dati riassuntivi di specificità, sensibilità e concordanza calcolati sui dati esposti nelle tabelle precedenti fra le metodiche GRIFOLS in comparazione con RADIM, Dade Behring, bioMériuex (Vidas ELFA), DELTA e GENELABS (WB).

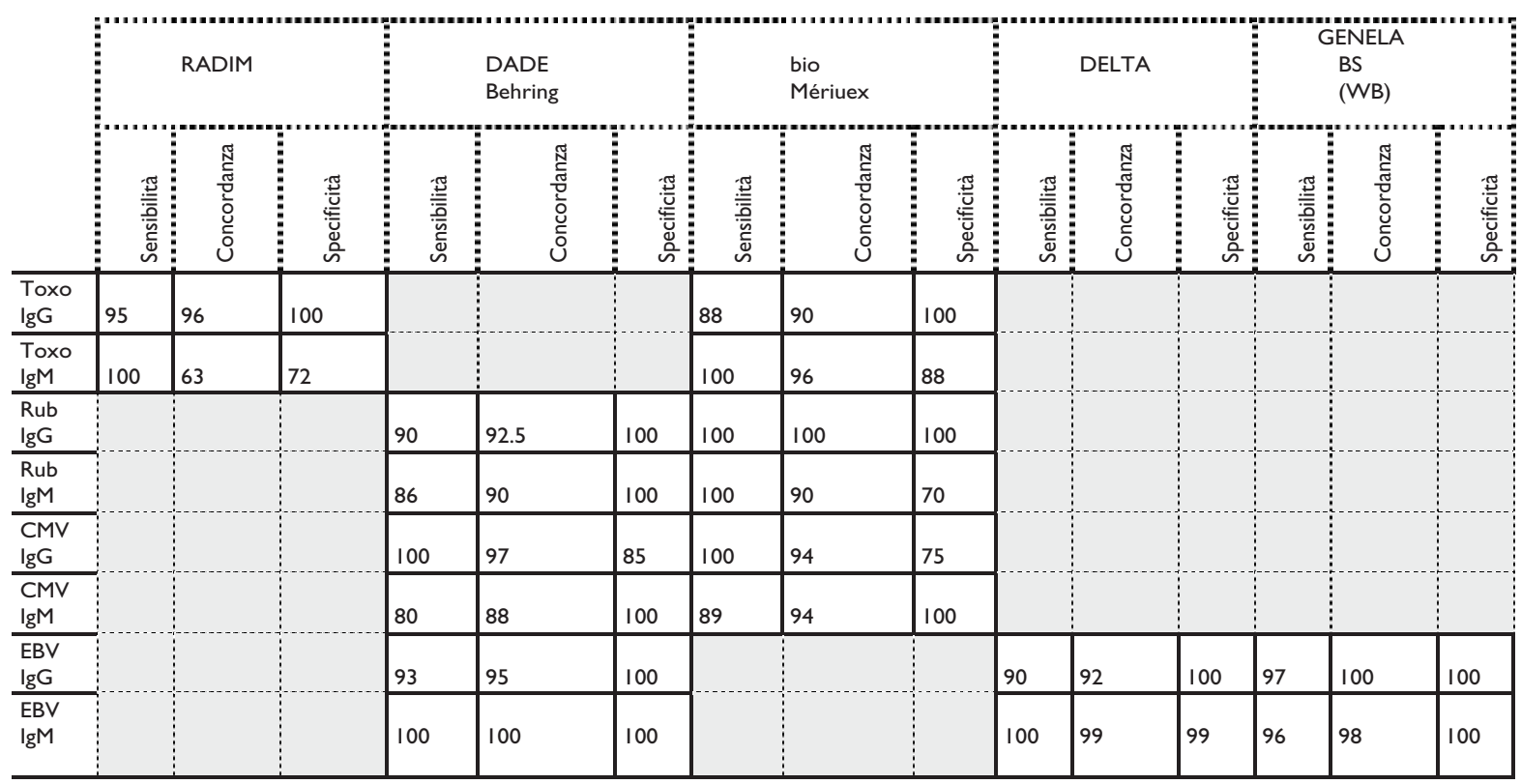

piastra): le differenze di sensibilità e specificità dimostrate fra i vari metodi impiegati sono assai limitate e sicuramente riconducibili a diversità antigeniche e di "coating" fra le varie preparazioni dei diversi produttori.

Un primo punto di valutazione concerne lo strumento Triturus che GRIFOLS propone come base dei tests diagnostici eseguiti in automazione. $\mathrm{Si}$ tratta di una macchina automatica semplice ed affidabile, capace di eseguire "in serie" un considerevole numero di analisi. Nel protocollo di studio impiegato per questa valutazione, il sistema automatizzato TRITURUS non è però mai stato portato a condizioni estreme di impiego, quali possono essere quelle imposte da sedute analitiche eseguite con il massimo numero di sieri analizzabili. È comunque possibile ipotizzare un buon comportamento del sistema, anche nelle condizioni estreme sopra descritte, dal momento che la performance del sistema si è dimostrata priva di vizi funzionali e strutturali e che il software gestionale è particolarmente semplice e comprensibile.

Il metodo SeraQuest-GRIFOLS ha dimostrato buona sensibilità per la determinazione delle IgG anti- T. gondii $(95 \%$ in paragone al test EIA di RADIM e $88 \%$ in confronto al metodo ELFAVIDAS), mentre è stata ottima la specificità del test $(100 \%$ rispetto ad entrambi i metodi di paragone). Per la determinazione delle IgM nei confronti di $T$. gondii i risultati sono stati, invece, ottimi per quanto riguarda la sensibilità $(100 \%$ verso entrambi i metodi di riferimento), mentre più scarsa è stata la specificità (da $72 \%$ a $88 \%$ ). Questi valori non sorprendono poiché, il metodo
SeraQuest-GRIFOLS, per quanto concerne la valutazione delle IgM, viene proposto come metodo di screening; i risultati falsamente positivi verranno individuati nel corso della prosecuzione dell'iter diagnostico mediante l'impiego di metodi dotati di maggiore specificità.

La determinazione della risposta anticorpale di classe IgG nei confronti del virus della Rosolia eseguita col metodo SeraQuest-GRIFOLS ha mostrato valori di sensibilità del $90 \%$ (rispetto ad Enzygnost-Dade Behring) e del 100\% (verso VIDAS - bioMériuex). La specificità calcolata nei confronti del test Enzygnost-Dade Behring e VIDAS-bioMériuex è stata pari al 100\%. Alla luce di questi dati il metodo SeraQuest può essere impiegato con successo nella diagnosi sierologica di stato immune verso il virus della Rosolia. Per quanto riguarda la ricerca di IgM, i valori di sensibilità calcolati verso i metodi Dade Behring e bioMérieux sono stati dell' $86 \%$ e $100 \%$, rispettivamente. I valori di specificità del metodo SeraQuest calcolati verso Dade Behring e VIDAS-bioMérieux sono stati del 100\% e del $70 \%$, rispettivamente. Anche questo dato non sorprende, in quanto il test ELFA viene considerato generalmente come "di conferma" e quindi dotato di buona specificità, mentre il test Enzygnost Dade Behring è considerato di screening (come il metodo SeraQuest - GRIFOLS).

Per quanto concerne la determinazione della risposta IgG nei confronti di CMV, la comparazione del metodo GRIFOLS con l'analogo test Dade Behring ha dimostrato valori di sensibilità del $100 \%$ e di specificità dell' $85 \%$. Quando il test SeraQuest è stato comparato con il metodo 
VIDAS i valori sono stati del $100 \%$ per la sensibilità e del $75 \%$ per la specificità, rispettivamente. La ricerca di anticorpi $\operatorname{IgM}$ mediante test SeraQuest-GRIFOLS ha mostrato, in paragone al metodo Enzygnost Dade Behring, una sensibilità dell' $80 \%$, mentre il confronto con il test VIDAS ha mostrato una sensibilità dell' $89 \%$; per entrambi i test a paragone la specificità del metodo GRIFOLS è stata del $100 \%$.

Pur non facendo parte del complesso ToRCH, il virus di EBV riveste una notevole importanza clinica per la sua grande diffusione e per il fatto di essere un'importante causa di linfoadenopatia che si manifesta sia nella forma di infezione primaria, la mononucleosi, sia in corso di linfoma EBV associato nel paziente immunodepresso. La mononucleosi infettiva, che colpisce il giovane adulto nella maggior parte dei casi, possiede caratteri laboratoristici (linfociti periferici attivati), clinici (linfoadenopatia, epatosplenomegalia, febbre, astenia) ed epidemiologici (età giovanile) che richiedono la diagnosi differenziale con patologie linfoproliferative gravate da prognosi assai peggiore. Pertanto le metodologie diagnostiche impiegate al fine di identificare l'infezione da EBV devono possedere notevole specificità per essere di utile impiego clinico. È questo il caso del metodo SeraQuest-GRIFOLS come evidenziato nel presente studio: la specificità calcolata è stata del 100\% (verso i metodi forniti da DELTA, Dade Behring e GENELABS WB) per la determinazione della risposta immune di classe $\mathrm{IgG}$, così come identici valori sono stati calcolati per la identificazione di anticorpi IgM, con la sola eccezione del confronto con il test EIA fornito da DELTA che ha mostrato un valore di specificità pari al 99\%. La sensibilità del test variava da un minimo di 96\% (verso GENELABS WB) al $100 \%$ (verso DELTA e Dade Behring).

Sono stati inoltre analizzati i risultati discrepanti identificati dai vari metodi impiegati nel caso di 34 sieri. Va precisato che da quest'analisi di concordanza sono stati esclusi i campioni che hanno dato risultati "border line" quando valutati con almeno tre delle metodiche usate in questo studio. I sieri sono stati quindi valutati come positivi o negativi sulla base della concordanza di risultato fra le differenti metodiche impiegate: è stato accettato come definitivo il risultato, positivo o negativo, che si ripeteva quando lo stesso campione era valutato con tre metodiche diverse.

Globalmente si evince come nel $35 \%$ dei casi, in termini di risultati, la metodica SeraQuest- GRIFOLS si sia dimostrata sovrapponibile alle altre metodiche valutate comparativamente in questo studio.

\section{BIBLIOGRAFIA}

1. Bruu AL, Hejetland R, Holter E, et al. Evaluation of 12 commecial tests for detection of Epstein Barr virus specific and heterophile antibodies. Clin diagn immunol 2000 may; 7(3): 451-6.

2. Decoster AL, Lambert N, Germaneau C, Masson C. "Toxoplasmosis serodiagnosis: comparison of Access TOXO IgMII assay compared to Axsim TOXO IgM and VIDAS TOXO IgM assay" Ann Biol Clin (Paris) 2000 nov-dic; 58 (6): 721-7.

3. Helfgott A. ToRCH testing in HIV-infected women. Clin Obstet Gynecol 1999; 42: 149-62.

4. Isada NB, Paar DP, Grossman JH, Straus SE. ToRCH infections. Diagnosis in the molecular age. J Reprod Med 1992; 37: 499-507.

5. Jenum PA, Stray-Pedersen B. Developments of specific immunoglobulins $\mathrm{G}, \mathrm{M}$, and A following primary Toxoplasma gondii infection in pregnant women. J Clin Microbiol 1998 oct; 36 (10): 2907-13.

6. Kanegane H, Nomura K, Miyawaki T, Tosato G. Biological aspects of Epstein Barr virus (EBV) infected lymphocytes in chronic active EBV infection and associated malignancies. Crit Rev Oncol Hematol 2002 dec; 44 (3): 239-49.

7. Leland D, French ML, Kleiman MB, Schreiner RL. The use of ToRCH titers. Pediatrics 1983; 72: 41-3.

8. Liesnard C, Donner C, Brancart F, Gosselin F, Delforge ML, Rodesch L. Prenatal diagnosis of congenital cytomegalovirus infetion: prospective study of 237 pregnancies at risk. Obstet Gynecol 2000 jun; 95 (6pt1): 881-8.

9. Montoya JG, Liesenfeld O, Kinney S, Press C, Remington JS. Vidas test for avidity of toxoplasma specific immunoglobulin $\mathrm{G}$ for confirmatory testing of pregnant women. J Clin Microbiol 2002 jul; 40 (7): $2504-8$

10. Newton ER. Diagnosis of perinatal TORCH infection. Clin Obstet Gynecol 1999 mar; 42 (1): 149-62.

11. Pujol-Rique M, Quinto L, Danes C, et al. Dating anti toxoplasma IgM in pregnancy using VIDAS ELFA methods. Enferm infecc microbiol 2000 jun; 18 (6): 274-8.

12. Sever JL, Ellenberg JH, Ley AC, Madden DL, Fuccillo DA, Tzan NR, Edmonds DM. Perinatal 'ToRCH' infections identified by serology: correlation with abnormalities in the children through 7 years of age. Int J Epidemiol 1992; 21: 285-92.

13. Stockman W, Bablock W, Poppe W, Bayer PM, Keller F, Schweiger CR. Evaluation Methods in Laboratory Medicine. Edited by Rainer Haeckel 1993.

14. Wilson AJ, Sant H, Van Duser P K, Wentz M. Enzyme-Based Methods for IgM Serology: Standard Indirect ELISA vs Antibody-Capture ELISA. Lab Med April 1992; 32 (4).

\section{Monica Sparacino}

UO Microbiologia settore batteriologia speciale e sierologia Ospedale S.Orsola

Via Massarenti, 9 - 40138 Bologna

Tel.: 051 4290909; e-mail: monicaspara@tiscali.it 